\title{
ADAMTS13 gene deletion enhances plasma high-mobility group box1 elevation and neuroinflammation in brain ischemia-reperfusion injury
}

Fujioka, Masayuki ; Nakano, Takafumi ; Hayakawa, Kazuhide ; Irie, Keiichi ; Akitake, Yoshiharu ; Sakamoto, Yuya ; Mishima, Kenichi ; Muroi, Carl ; Yonekawa, Yasuhiro ; Banno, Fumiaki ; Kokame, Koichi ; Miyata, Toshiyuki ; Nishio, Kenji ; Okuchi, Kazuo ; Iwasaki, Katsunori ; Fujiwara, Michihiro ; Siesjö, Bo K

\begin{abstract}
Highly adhesive glycoprotein von Willebrand factor (VWF) multimer induces platelet aggregation and leukocyte tethering or extravasation on the injured vascular wall, contributing to microvascular plugging and inflammation in brain ischemia-reperfusion. A disintegrin and metalloproteinase with thrombospondin type-1 motifs 13 (ADAMTS13) cleaves the VWF multimer strand and reduces its prothrombotic and proinflammatory functions. Although ADAMTS13 deficiency is known to amplify postischemic cerebral hypoperfusion, there is no report available on the effect of ADAMTS13 on inflammation after brain ischemia. We investigated if ADAMTS13 deficiency intensifies the increase of extracellular HMGB1, a hallmark of post-stroke inflammation, and exacerbates brain injury after ischemia-reperfusion. ADAMTS13 gene knockout (KO) and wild-type (WT) mice were subjected to 30-min middle cerebral artery occlusion (MCAO) and 23.5-h reperfusion under continuous monitoring of regional cerebral blood flow (rCBF). The infarct volume, plasma high-mobility group box1 (HMGB1) level, and immunoreactivity of the ischemic cerebral cortical tissue (double immunofluorescent labeling) against HMGB1/NeuN (neuron-specific nuclear protein) or HMGB1/MPO (myeloperoxidase) were estimated 24h after MCAO. ADAMTS13KO mice had larger brain infarcts compared with WT $24 \mathrm{~h}$ after MCAO $(\mathrm{p}<0.05)$. The rCBF during reperfusion decreased more in ADAMTS13KO mice. The plasma HMGB1 increased more in ADAMTS13KO mice than in WT after ischemia-reperfusion $(\mathrm{p}<0.05)$. Brain ischemia induced more prominent activation of inflammatory cells co-expressing HMGB1 and MPO and more marked neuronal death in the cortical ischemic penumbra of ADAMTS13KO mice. ADAMTS13 deficiency may enhance systemic and brain inflammation associated with HMGB1 neurotoxicity, and aggravate brain damage in mice after brief focal ischemia. We hypothesize that ADAMTS13 protects brain from ischemia-reperfusion injury by regulating VWF-dependent inflammation as well as microvascular plugging
\end{abstract}

DOI: https://doi.org/10.1007/s10072-011-0913-9

Posted at the Zurich Open Repository and Archive, University of Zurich

ZORA URL: https://doi.org/10.5167/uzh-156028

Journal Article

Published Version

Originally published at:

Fujioka, Masayuki; Nakano, Takafumi; Hayakawa, Kazuhide; Irie, Keiichi; Akitake, Yoshiharu; Sakamoto, Yuya; Mishima, Kenichi; Muroi, Carl; Yonekawa, Yasuhiro; Banno, Fumiaki; Kokame, Koichi; Miyata, Toshiyuki; Nishio, Kenji; Okuchi, Kazuo; Iwasaki, Katsunori; Fujiwara, Michihiro; Siesjö, Bo K (2012). 
ADAMTS13 gene deletion enhances plasma high-mobility group box1 elevation and neuroinflammation in brain ischemia-reperfusion injury. Neurological Sciences, 33(5):1107-1115.

DOI: https://doi.org/10.1007/s10072-011-0913-9 


\title{
ADAMTS13 gene deletion enhances plasma high-mobility group box1 elevation and neuroinflammation in brain ischemia-reperfusion injury
}

\author{
Masayuki Fujioka $\cdot$ Takafumi Nakano $\cdot$ Kazuhide Hayakawa Keiichi Irie $\cdot$ Yoshiharu Akitake \\ Yuya Sakamoto $\cdot$ Kenichi Mishima $\cdot$ Carl Muroi • Yasuhiro Yonekawa · Fumiaki Banno • \\ Koichi Kokame $\cdot$ Toshiyuki Miyata Kenji Nishio $\cdot$ Kazuo Okuchi $\cdot$ Katsunori Iwasaki • \\ Michihiro Fujiwara $\cdot$ Bo K. Siesjö
}

Received: 13 August 2011/Accepted: 20 December 2011/Published online: 3 January 2012

(c) Springer-Verlag 2011

\begin{abstract}
Highly adhesive glycoprotein von Willebrand factor (VWF) multimer induces platelet aggregation and leukocyte tethering or extravasation on the injured vascular wall, contributing to microvascular plugging and inflammation in brain ischemia-reperfusion. A disintegrin and metalloproteinase with thrombospondin type-1 motifs 13 (ADAMTS13) cleaves the VWF multimer strand and reduces its prothrombotic and proinflammatory functions. Although ADAMTS13 deficiency is known to amplify
\end{abstract}

M. Fujioka $(\bowtie) \cdot$ T. Nakano $\cdot$ K. Hayakawa $\cdot$ K. Irie

Y. Akitake · Y. Sakamoto · K. Mishima - C. Muroi ·

K. Iwasaki · M. Fujiwara

Department of Neuropharmacology, Faculty of Pharmaceutical

Sciences, Fukuoka University, Fukuoka, Japan

e-mail: mfujioka_2000_99@yahoo.co.jp

M. Fujioka

Stroke Center, Helios General Hospital Aue, Dresden University

of Technology, Dresden, Saxony, Germany

M. Fujioka $\cdot$ C. Muroi $\cdot$ Y. Yonekawa

Department of Neurosurgery, University of Zurich,

Zurich, Switzerland

M. Fujioka $\cdot$ K. Nishio $\cdot$ K. Okuchi

Emergency and Critical Care Medical Center,

Nara Medical University, Nara, Japan

K. Irie $\cdot$ Y. Akitake $\cdot$ K. Mishima $\cdot$ C. Muroi - K. Iwasaki Institute for Aging and Brain Sciences,

Fukuoka University, Fukuoka, Japan

F. Banno $\cdot$ K. Kokame $\cdot$ T. Miyata

Research Institute, National Cerebral

and Cardiovascular Center, Suita, Japan

B. K. Siesjö

Laboratory for Experimental Brain Research,

Lund University, Lund, Sweden post-ischemic cerebral hypoperfusion, there is no report available on the effect of ADAMTS13 on inflammation after brain ischemia. We investigated if ADAMTS13 deficiency intensifies the increase of extracellular HMGB1, a hallmark of post-stroke inflammation, and exacerbates brain injury after ischemia-reperfusion. ADAMTS13 gene knockout (KO) and wild-type (WT) mice were subjected to 30-min middle cerebral artery occlusion (MCAO) and 23.5-h reperfusion under continuous monitoring of regional cerebral blood flow (rCBF). The infarct volume, plasma high-mobility group box1 (HMGB1) level, and immunoreactivity of the ischemic cerebral cortical tissue (double immunofluorescent labeling) against HMGB1/NeuN (neuron-specific nuclear protein) or HMGB1/MPO (myeloperoxidase) were estimated $24 \mathrm{~h}$ after MCAO. ADAMTS $13 \mathrm{KO}$ mice had larger brain infarcts compared with WT $24 \mathrm{~h}$ after MCAO $(p<0.05)$. The rCBF during reperfusion decreased more in ADAMTS13KO mice. The plasma HMGB1 increased more in ADAMTS13KO mice than in WT after ischemia-reperfusion $(p<0.05)$. Brain ischemia induced more prominent activation of inflammatory cells co-expressing HMGB1 and MPO and more marked neuronal death in the cortical ischemic penumbra of ADAMTS13KO mice. ADAMTS13 deficiency may enhance systemic and brain inflammation associated with HMGB1 neurotoxicity, and aggravate brain damage in mice after brief focal ischemia. We hypothesize that ADAMTS13 protects brain from ischemia-reperfusion injury by regulating VWF-dependent inflammation as well as microvascular plugging.

Keywords Brain ischemia-reperfusion .

High-mobility group box1 - ADAMTS13 .

Inflammation - Von Willebrand factor .

Thrombotic thrombocytopenic purpura 


\section{Introduction}

The post-ischemic inflammation incites stroke evolution [15, 16, 19-21, 32]. An ischemic insult triggers leukocytes infiltration and astrocyte and microglia activations in the affected brain, leading to the increase of the high-mobility group box1 (HMGB1) in the plasma and brain of the stroke model $[19,21]$. The HMGB1 is a potent proinflammatory cytokine secreted by blood-immune [23, 29, 45] and brainglial cells [35]. This extracellular HMGB1 further activates monocytes/macrophages [3, 34], neutrophils [1, 34], microvascular endothelial cells [12], astrocytes [36] and microglia [24], amplifies the systemic and brain inflammation, and extends the ischemic brain damage into the penumbra [19, 21, 24, 27, 33, 38].

Within the ischemic brain vasculature after middle cerebral artery occlusion (MCAO), circulating platelets [2, 28] and leukocytes [9, 28, 31] are activated, inducing microvascular obstructions and inflammation. In the initial activations of platelet and leukocyte on ischemic endothelium, a large multimeric adhesive glycoprotein von Willebrand factor (VWF) plays a central role. The VWF multimer tethers platelets on the vascular endothelial surface, leading to platelet activation [40]. This platelet-decorated VWF multimer string bound to endothelium supports leukocytes tethering, rolling and transmigration on stimulated vascular endothelial cells, and links thrombosis to inflammation [5, 7, 37]. The platelet binding affinity of VWF increases with increasing length of the VWF multimer strand and with high fluid shear stress [40, 44]. Accordingly, the longest multimer termed ultra-large VWF (ULVWF; secreted by vascular endothelium upon stimulation) exerts its maximum prothrombotic and proinflammatory functions in the microvasculture or stenotic vessels under high shear stress condition [30, 40, 41, 43].

A disintegrin and metalloproteinase with thrombospondin type-1 motifs 13 (ADAMTS13) inhibits these VWF functions by cleaving the Tyr1605-Met1606 bond in the A2 domain of the VWF [13, 41]. Physiologically, circulating ADAMTS13 cleaves the ULVWF secreted from endothelial cells, releasing tethered platelets and VWF fragments [11]. In a setting of on-going thrombus formation, the high shear stress induced at the stenotic vasculature stretches plasma-derived VWF multimers (smaller than ULVWF) on the thrombus surface. The extended VWF multimers involved in the platelet thrombosis are consequently cleaved by ADAMTS13 [41]. Notably, by decreasing the interaction between the ULVWF-platelet strands and leukocytes, ADAMTS13 reduces leukocytes adhesion and extravasation on the stimulated vascular wall and down-regulates tissue inflammation [5, 7, 37]. ADAMTS13 deficiency in humans increases the circulating ULVWF resulting in thrombotic thrombocytopenic purpura
(TTP) $[17,41]$. The TTP manifests fever and neurological deficits associated with VWF-platelet microthrombus formation in the brain. This implies that ADAMTS13 plays a role in inflammation after brain ischemia in TTP patients.

Early studies have shown that ADAMTS13 deficiency aggravates ischemic brain damage in experimental stroke models $[14,48]$. We revealed that in the ADAMTS13deficient mice after a brief focal ischemia the post-ischemic hypoperfusion was significantly amplified partly because of enhanced microvascular plugging by VWF-platelet-leukocyte complex [14]. However, it still remains unclear if an enhanced inflammatory reaction is involved in the deterioration of ischemic brain injury under ADAMTS13 deficiency. Here, we investigated whether ADAMTS13 gene deletion intensifies the increase of extracellular HMGB1, a hallmark of post-stroke inflammation, and exacerbates the brain damage after ischemia-reperfusion.

\section{Materials and methods}

\section{Animals}

The effect of ADAMTS13 gene deletion on inflammation after brain ischemia was investigated using male ADAMTS13KO and littermate WT mice in an SV129 genetic background, originally generated as a TTP model by our study group [4]. Studies using KO $(n=31)$ and WT $(n=31)$ mice $(8-10$ weeks of age, $20-23 \mathrm{~g}$ of body weight) were approved by the institutional ethics committee. The genotype of each animal was kept unspecified until all experiment's completion.

\section{Middle cerebral artery occlusion}

Thirty-minute MCAO by thread insertion from the common carotid artery was induced in $\mathrm{KO}(n=21)$ and WT $(n=21)$ mice as previously described [14, 19-21]. Mice were anesthetized with $2 \%$ halothane for induction and maintained on $1 \%$ halothane in $70 \% \mathrm{~N}_{2} \mathrm{O}$ and $30 \% \mathrm{O}_{2}$ by face mask. Body temperature was maintained at 36.5$37.0^{\circ} \mathrm{C}$ during surgery. Successful left MCAO was confirmed according to the following criteria: (1) rCBF in the left cerebral cortex at the thread insertion less than $20 \%$ of the pre-MCAO $\mathrm{rCBF}$, and (2) consistent presence of significant ischemic neurological symptoms of the left cerebral hemisphere, characterized by right paresis and right circling behavior, during 30-min MCAO. The MCAO surgery was performed within 7 min without bleeding. The anesthesia was discontinued during 30-min MCAO. There were no statistically significant differences in body temperature between the two groups immediately before or after the thread insertion, or 10, 20, and $30 \mathrm{~min}$ after 
MCAO. The thread was removed under re-anesthesia after 30-min MCAO. Sham surgery in KO $(n=10)$ and WT $(n=10)$ involved temporary insertion $(1 \mathrm{~s})$ of the thread into the left common carotid artery without MCAO. There were no statistical differences in prothrombin time or survival rate between $\mathrm{KO}$ and WT mice at $24 \mathrm{~h}$ after MCAO (Table 1).

Regional cerebral blood flow

The rCBF was measured by laser Doppler flowmetry (LDF) (ALF21, Advance Co., Tokyo, Japan) as previously described [14, 20]. The LDF probe was placed through a guide cannula into the left cerebral cortex stereotaxically (0.22 $\mathrm{mm}$ posterior and $2.5 \mathrm{~mm}$ lateral from bregma; $1.5 \mathrm{~mm}$ depth from the skull surface) on a stereotaxic instrument (Narishige Scientific Instrument Lab: SR-5 M, Tokyo) under anesthesia (pentobarbital $50 \mathrm{mg} / \mathrm{kg}$, i.p.) $24 \mathrm{~h}$ before MCAO or sham surgery. The rCBF was monitored in all animals continuously from $30 \mathrm{~min}$ before MCAO until immediately after reperfusion. In randomly selected animals, the rCBF was repeatedly recorded over time within $24 \mathrm{~h}$ after MCAO. The $\mathrm{rCBF}$ during occlusion and reperfusion was expressed as percentages of the preMCAO LDF baseline value.

Infarct volume and neurological deficit

The brains were sectioned coronally (four 2-mm thick slices) according to a mouse brain matrix $24 \mathrm{~h}$ after MCAO (KO, $n=14$ and WT, $n=15$ ) or sham operation (KO, $n=5$ and WT, $n=5$ ). The infarct area was measured in each slice stained with 2,3,5 triphenyltetrazolium chloride with an image analysis system (NIH Image, version 1.63), and the infarct volume was calculated [14, 19-21]. Neurological deficit score ranging from 0 (normal motor function) to 5 (no spontaneous motor activity) was measured at $24 \mathrm{~h}$ after MCAO [14, 19, 21] (Table 1).
Plasma HMGB1 measurement

The plasma HMGB1 protein was evaluated by western blot $24 \mathrm{~h}$ after MCAO. Plasma samples were fractionated by sodium dodecyl sulfate (SDS)-polyacrylamide gel electrophoresis, and HMGB1 levels were determined by immunoblotting with respect to a standard curve, with recombinant HMGB1 as a reference (Sigma-Aldrich, Tokyo) $[19,21]$. A blood sample $(500 \mu \mathrm{L})$ was taken from each experimental animal via inferior vena cava $24 \mathrm{~h}$ after MCAO (KO, $n=11$ and WT, $n=12$ ) or sham surgery (KO, $n=10$ and WT, $n=10$ ). The sample was centrifuged $\left(3,000 \mathrm{rpm}\right.$ at $4^{\circ} \mathrm{C}$ for $\left.10 \mathrm{~min}\right)$, and the supernatant $(200 \mu \mathrm{L})$ was further centrifuged $\left(15,000 \mathrm{rpm}\right.$ at $4{ }^{\circ} \mathrm{C}$ for $20 \mathrm{~min})$. SDS sample buffer $[125 \mathrm{mmol} / \mathrm{L}$ Tris $(\mathrm{pH} 6.8)$, $2 \%$ SDS, $20 \%$ glycerol, $0.0001 \%$ bromophenol blue, and $10 \% \beta$-mercaptoethanol] $(100 \mu \mathrm{L})$ was added to the plasma extract solution $(100 \mu \mathrm{L})$, and the resultant sample was heated at $95^{\circ} \mathrm{C}$ for $5 \mathrm{~min}$. Protein $(15 \mu \mathrm{g})$ was separated by SDS-polyacrylamide gel electrophoresis (20\% gel). Blotting was performed at $2 \mathrm{~mA} / \mathrm{cm}^{2}$ by semi-dry type blotting (Bio-Rad, Tokyo, Japan). The blots were blocked with 5\% non-fat dry milk in Tris-buffered saline in $0.1 \%$ Tween 20 (TBS-T) at $4{ }^{\circ} \mathrm{C}$ and incubated with goat polyclonal antiHMGB1 primary antibody (1:200) (Santa Cruz Biotechnology, Santa Cruz, CA, USA) in TBS-T, followed by bovine anti-goat IgG (heavy chain and light chain $[\mathrm{H}+\mathrm{L}])$ alkaline phosphatase conjugate (1:1000) in TBS-T. The blots were visualized with the use of alkaline phosphatase color reagents. The signal intensity of the blots was measured with an image analysis system (NIH Image, version 1.63).

\section{Double immunohistochemical staining for HMGB1/NeuN and HMGB1/MPO}

Double immunofluorescent labeling for HMGB1 with NeuN or MPO on paraffin-embedded 5- $\mu$ m coronal sections

Table 1 Effect of ADAMTS13 gene deletion on brain ischemia in mice after 30-min MCAO and 23.5-h reperfusion

\begin{tabular}{llll}
\hline & WT & & KO \\
\hline Brain infarct volume $\left(\mathrm{mm}^{3}\right)$ & $12.0 \pm 2.0(n=15)$ & $p<0.05^{\mathrm{a}}$ & $28.5 \pm 5.8(n=14)$ \\
Neurological deficit score & $1.3 \pm 0.2(n=15)$ & $p<0.05^{\mathrm{a}}$ & $1.9 \pm 0.2(n=14)$ \\
Prothrombin time $(\mathrm{s})$ & $12.1 \pm 0.8(n=7)$ & $\mathrm{ns}$ & $11.6 \pm 0.9(n=6)$ \\
Survival rate $(\%)$ & $95.2(n=20 / 21)$ & $\mathrm{ns}$ & $90.4(n=19 / 21)$ \\
\hline
\end{tabular}

The values are expressed as the mean \pm SEM. Neurological deficit score, score 0 ; normal motor function, 1 ; flexion of torso and of contralateral forelimb upon lifting of the animal by the tail, 2; circling to the ipsilateral side but normal posture at rest, 3; circling to the ipsilateral side, 4; rolling to the ipsilateral side, 5; leaning to the ipsilateral side at rest (no spontaneous motor activity)

$n s$ Statistically not significant

a Student $t$ test 
of the mouse brain was analyzed by fluorescence microscopy (Nikon, AZ-FL, Tokyo, Japan). At $24 \mathrm{~h}$ after MCAO (KO, $n=5$; WT, $n=5$ ) or sham surgery (KO, $n=5$; WT, $n=5$ ), mice were humanely perfused transcardially with saline and $4 \%$ paraformaldehyde. The brains were removed of fat and water using an autodegreasing unit (RH-12; Sakura Seiko Co, Tokyo) and embedded in paraffin. Subsequently, $5-\mu \mathrm{m}$ sections were mounted on slides and dried at $37^{\circ} \mathrm{C}$ for 1 day. After deparaffinization and rehydration, the sections were incubated with primary antibodies of biotinylated anti-mouse NeuN (1:200; Chemicon International, Temecula, CA, USA) or rabbit polyclonal anti-MPO (1:200; DAKO Inc., Carpinteria, CA, USA) and of goat polyclonal anti-HMGB1 (1:200; Santa Cruz Biotechnology, Santa Cruz, CA, USA) overnight at $4^{\circ} \mathrm{C}$. Sections were then incubated with donkey anti-goat IgG-FITC secondary antibody (1:200; Santa Cruz Biotechnology) for $1 \mathrm{~h}$, and thereafter with goat anti-rabbit IgG-Texas red secondary antibody (1:200; Santa Cruz Biotechnology) or Ultra avidine Texas Red (1:200; Leinco Technologies) for $1 \mathrm{~h}$. The sections were imaged and analyzed. The histological findings were evaluated by neuropathologists until a consensus was obtained. The fluorescence intensity (for cells positive to NeuN, MPO, or HMGB1) in five randomly selected areas $(150 \mu \mathrm{m} \times 200 \mu \mathrm{m}$ for each) from the region of interest in the ischemic cerebral cortex (as indicated in Figs. 2,3) was evaluated with an image analysis system (NIH Image, version 1.63) with the corresponding non-ischemic contralateral regions as a control, and the relative fluorescence intensity was calculated. In ischemic stroke, the necrotic core is surrounded by a zone of reactive/inflammatory cytosis which can extend the initial insult into the penumbra with delayed cell death. Based on this concept, the region of interest in the cortical penumbra for the fluorescence evaluation was decided as indicated.

\section{Statistical analysis}

Data are presented as mean \pm standard error of mean (SEM). For multiple pairwise comparisons in parametric analysis, two-way analysis of variance (ANOVA) followed by Tukey-Kramer's test was performed. When only two groups were compared, Student's $t$ test was used. Probability values of $<0.05$ were considered statistically significant.

\section{Results}

Brain infarction

The ADAMTS13 gene knockout (ADAMTS13KO) mice group had a significantly larger volume of brain infarction compared with the wild-type (WT) following 23.5-h reperfusion after 30-min MCAO (Student's $t$ test) (Table 1). No ischemic brain damage was observed in either $\mathrm{KO}$ or WT mice after sham operation.

Neurological deficits

ADAMTS13KO mice had more severe neurological deficits than the WT (Student's $t$ test) $24 \mathrm{~h}$ after MCAO (Table 1).

Regional cerebral blood flow

The rCBF showed no statistical differences between the two groups during MCAO or immediately after reperfusion. However, the rCBF in ADAMTS13KO mice progressively decreased significantly more markedly compared to WT during the first 30-min reperfusion (Tukey-Kramer's test). $24 \mathrm{~h}$ after MCAO, the rCBF in KO mice remained significantly lower compared to WT (Student's $t$ test) (Table 2).

ADAMTS13 gene deletion enhances post-ischemic increase of plasma HMGB1

A 30-min MCAO and 23.5-h reperfusion significantly increased the plasma HMGB1 level both in ADAMTS13KO and WT mice as compared to sham operation (Fig. 1). However, this increase of plasma HMGB1 after MCAO was more markedly enhanced in ADAMTS13KO mice than in WT (Tukey-Kramer's test).

ADAMTS13 gene deletion intensifies post-ischemic brain inflammation and neuronal death

\section{Double immunohistochemical staining for HMGB1/NeuN} and $H M G B 1 / M P O$

The qualitative analysis of double immunofluorescent labeling for HMGB1 with NeuN (neuron-specific nuclear protein) or MPO (myeloperoxidase) on the cortical tissue $24 \mathrm{~h}$ after MCAO or sham operation showed that (1) the number of neurons immunoreactive to NeuN in the ischemic penumbra decreased more in ADAMTS13KO mice compared to WT (Fig. 2), (2) HMGB1 immunoreactivity disappeared in the ischemic neuronal nuclei in both $\mathrm{KO}$ and WT mice, suggestive of a translocation of HMGB1 from neuronal nucleus either to neuronal cytoplasm or to extracellular space (Fig. 2), and (3) cells with co-expression of MPO (a marker for neutrophils, macrophages and/ or microglia) and HMGB1 appeared more prominently in the ischemic penumbra in ADAMTS13KO mice than in WT (Fig. 3). 
Table 2 Effect of ADAMTS13 gene deletion on regional cerebral blood flow (rCBF) in mice of 30-min MCAO model

\begin{tabular}{|c|c|c|c|}
\hline & \multicolumn{2}{|l|}{$\mathrm{WT}(n=6)$} & $\mathrm{KO}(n=7)$ \\
\hline \multicolumn{4}{|l|}{ Time (min) } \\
\hline Baseline & $100.0 \pm 0$ & ns & $100.0 \pm 0$ \\
\hline 0 & $13.6 \pm 4.6$ & ns & $13.2 \pm 2.0$ \\
\hline 10 & $17.9 \pm 3.4$ & $\mathrm{~ns}$ & $22.4 \pm 6.3$ \\
\hline 20 & $17.7 \pm 5.2$ & ns & $19.3 \pm 5.7$ \\
\hline 30 & $16.8 \pm 4.7$ & $\mathrm{~ns}$ & $18.6 \pm 3.5$ \\
\hline Reperfusion & $114.3 \pm 19.1$ & $\mathrm{~ns}$ & $88.1 \pm 8.1$ \\
\hline 40 & $90.5 \pm 12.7$ & $p<0.05^{\mathrm{a}}$ & $48.4 \pm 8.4$ \\
\hline 50 & $93.8 \pm 11.3$ & $p<0.01^{\mathrm{a}}$ & $35.4 \pm 7.7$ \\
\hline 60 & $83.2 \pm 6.8$ & $p<0.01^{\mathrm{a}}$ & $28.1 \pm 4.7$ \\
\hline \multicolumn{4}{|l|}{ Time (hour) } \\
\hline 24 & $72.9 \pm 13.9$ & $p<0.05^{\mathrm{b}}$ & $37.2 \pm 5.9$ \\
\hline
\end{tabular}

Relative fluorescent intensity of NeuN, MPO and HMGBI

The relative fluorescent intensity (\%) in the ischemic cerebral cortex $24 \mathrm{~h}$ after MCAO was significantly decreased for NeuN in ADAMTS13KO mice compared to WT $[57.5 \pm 8.7$ in KO vs. $93.2 \pm 5.5$ in WT $(p<0.05$, student's $t$ test)], and increased for both MPO and HMGB1 more in ADAMTS13KO mice than in WT [KO vs. WT: $557.7 \pm 139.8$ vs. $310.4 \pm 131.7$ for MPO, and $159.7 \pm$ 46.5 vs. $70.2 \pm 35.1$ for HMGB1 (although not statistically significant)].

\section{Discussion}

The (UL)VWF, the substrate of ADAMTS13, recruits platelets and leukocytes onto the injured vascular endothelium, and mediates microvascular plugging and enhances the tissue inflammation [5, 7, 30, 37, 40, 41, 43, 44]. ADAMTS13 inhibits these prothrombotic and proinflammatory functions of (UL)VWF [7, 13, 41]. Our current study implies that ADAMTS13 gene deletion amplifies systemic and brain inflammatory responses against brain ischemia-reperfusion enhancing a potent cytokine HMGB1 neurotoxicity, leads to progressive decline of post-ischemic cerebral blood reflow, and exacerbates ischemic brain injury. ADAMTS13 may play a neuroprotective role against inflammation in ischemic stroke.

ADAMTS13 deficiency may promote inflammation by activating platelets, leukocytes, and vascular endothelium after brain ischemia-reperfusion. Responding to ischemia-

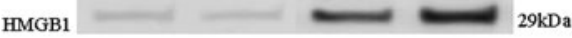

Sham-WT Sham-KO MCAO-WT MCAO-KO

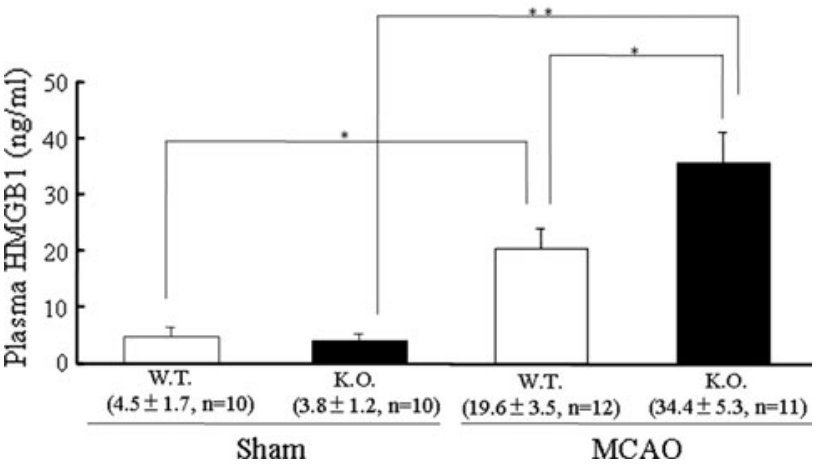

Fig. 1 Effect of ADAMTS13 gene deletion on plasma HMGB1 in mice after 30-min MCAO. The plasma HMGB1 protein was evaluated by western blot. Transient focal ischemia of $30-\mathrm{min}$ MCAO followed by 23.5-h reperfusion significantly increased the plasma HMGB1 level both in ADAMTS13KO and WT mice when compared to sham operation [plasma HMGB1 (ng/ml): MCAO-KO; $34.4 \pm 5.3$ vs. sham-KO; $3.8 \pm 1.2, p<0.01$, and MCAO-WT; $19.6 \pm 3.5$ vs. sham-WT; $4.5 \pm 1.7, p<0.05$, Tukey-Kramer's test after two-way ANOVA $(F(1,40)=38.401, p<0.01)]$. This increase of plasma HMGB 1 at $24 \mathrm{~h}$ after MCAO was more markedly enhanced in ADAMTS13KO mice than in WT [MCAO-KO; $34.4 \pm 5.3$ vs. MCAO-WT; $19.6 \pm 3.5, p<0.05$, Tukey-Kramer's test after twoway ANOVA $(F(1,40)=4.296, p<0.05)]$. Sham-WT $n=10$, Sham-KO $n=10$, MCAO-WT $n=12$, MCAO-KO $n=11$. Values are expressed as the mean \pm SEM. ${ }^{*} p<0.05$, ${ }^{*} p<0.01$, TukeyKramer's test after two-way ANOVA

reperfusion, the stimulated vascular endothelial cells secrete ULVWF [44]. Binding of VWF to the platelet membrane glycoprotein initiates a signaling cascade that causes platelet activation [25, 46]. The activated platelets release multiple proinflammatory factors, mitogenic mediators, metalloproteinases, and reactive oxygen species, and stimulate the leukocytes and endothelium to incite inflammatory reactions $[8,10]$. Further, the platelet(UL)VWF string directly supports the leukocyte transmigration into the inflammatory tissue $[5,7,37]$. Thus, the VWF-cleaving protease ADAMTS13 plays a role as an anti-inflammatory factor, and therefore its deficiency can exaggerate the post-ischemic inflammation.

In ADAMTS13KO mice after a cerebral ischemia, the plasma HMGB1 increased more and the HMGB1expressing immune cells appeared more prominent in the cortical penumbra than in WT. The increased extracellular HMGB1 may contribute to secondary ischemic brain damage in ADAMTS13KO mice. The HMGB1, a DNAbinding protein, is a central proinflammatory cytokine [29]. Upon inflammatory signals, the chromosomal HMGB1 relocates into cytoplasmic secretory lysosomes and is secreted into the immunological synapse [29] or into the extracellular space by monocytes/macrophages [45], neutrophils [23], mature dendritic cells [29], natural killer cells 


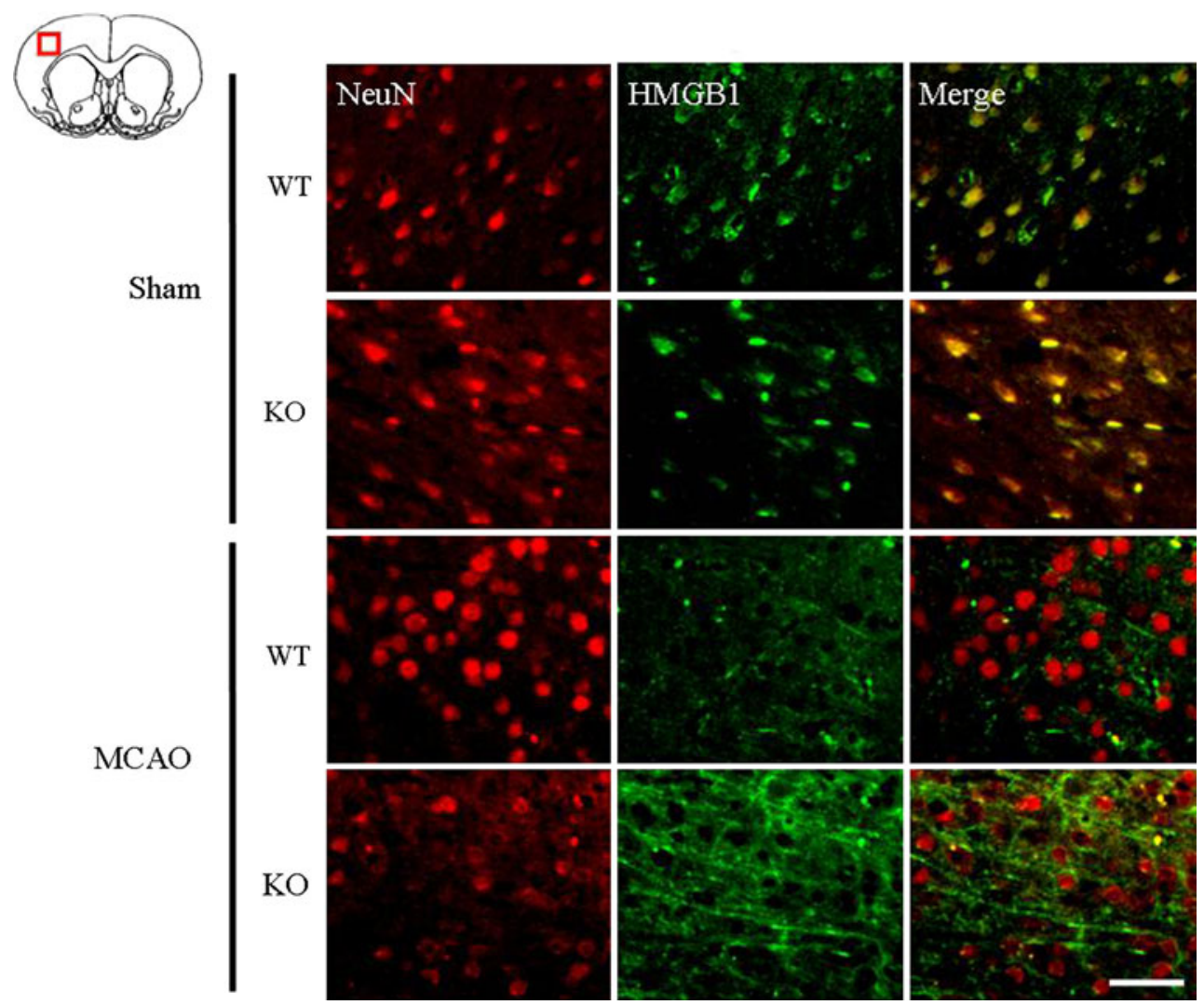

Fig. 2 Effect of ADAMTS13 gene deletion on NeuN positive cells expressing HMGB1 in mice brain after 30-min MCAO. Qualitative analysis of double immunofluorescent labeling for HMGB1 with NeuN on the brain tissue $24 \mathrm{~h}$ after MCAO showed that the number of neurons immunoreactive to NeuN in the ischemic cortical penumbra decreased more in ADAMTS13KO mice than WT. The HMGB1 immunoreactivity disappeared in the ischemic neuronal nuclei in both KO and WT mice, suggesting that neuronal-nuclear HMGB1

[29], and glia [35]. HMGB1 also leaks from necrotic cells [42] and ischemic neurons [38]. The extracellular HMGB1 binds to its receptors, RAGE (receptor for advanced glycation end products) [22], Toll-like receptor 2 (TLR2), and TLR4 [34], recapitulates the intracellular signaling cascades initiated by early proinflammatory stresses, and thus propagates continuous proinflammatory responses $[3,22$, 29, 34, 42]. Naturally, high blood HMGB1 correlates with the severity of systemic inflammation [29, 45].

After brain ischemia, extracellular HMGB1 increases rapidly in the blood and central nervous system, and induces neuroinflammation [24, 38]. HMGB-1 early released from the striatal ischemic core can bind to RAGE that is robustly expressed in the peri-infarct region, and extend the ischemic brain injury [38]. HMGB1-RAGE signaling in infiltrating macrophages and activated microglia seemingly mediate neuronal death evolution in the ischemic penumbra [33]. The HMGB1 inhibition in the brain or systemic circulation protects the blood-brain barrier and the brain from ischemia [24, 27, 33, 47]. translocated into either the neuronal cytoplasm or the extracellular space. In addition, the HMGB1 immunoreactivity seemed to increase in the ischemic cortical tissue in the ADAMTS13KO mice, indicating a possibility that non-neuronal HMGB1 positive cells were activated in the KO mice compared to the WT ( $n=5$ in each group). Scale bar $50 \mu \mathrm{m}$. NeuN positive cells red, HMGB1 positive cells green, merge yellow

Inhibition of leukocytes and microglia results in decreased HMGB1 levels in the brain and plasma, reduces apoptosis in the ischemic brain, and improves brain atrophy and neurologic deficits [19, 21]. Therefore, the increased extracellular HMGB1 in the blood and brain of ADAMTS13KO mice as observed in our study can exacerbate ischemic brain injury by intensifying systemic and brain inflammation. Interestingly, the platelet intracellular HMGB1 is exported to the external surface of the plasma membrane upon its activation [39]. Accordingly, the activated platelet may be an additional source of the exceedingly increased plasma HMGB1 in ADAMTS13KO mice after brain ischemia, where enhanced VWF-platelet interactions develop [14]. We suggest that ADAMTS13 deficiency multiplies secondary insults after brain ischemia by up-regulating (UL)VWF-mediating inflammation and enhancing HMGB1 neurotoxicity in the systemic and local environments.

This study suggests a potential therapy with ADAMTS13 for acute ischemic stroke by breaking a vicious circle 
Fig. 3 Effect of ADAMTS13 gene deletion on MPO positive cells expressing HMGB1 in mice brain after 30-min MCAO. Qualitative analysis of double immunofluorescent labeling for HMGB1 with MPO on the brain tissue $24 \mathrm{~h}$ after MCAO showed that cells co-expressing MPO and HMGB1 were more prominent in the ischemic cortical penumbra in $\mathrm{KO}$ mice than in WT ( $n=5$ in each group). Scale bar $50 \mu \mathrm{m}$. MPO positive cells red, HMGB1 positive cells green, merge yellow

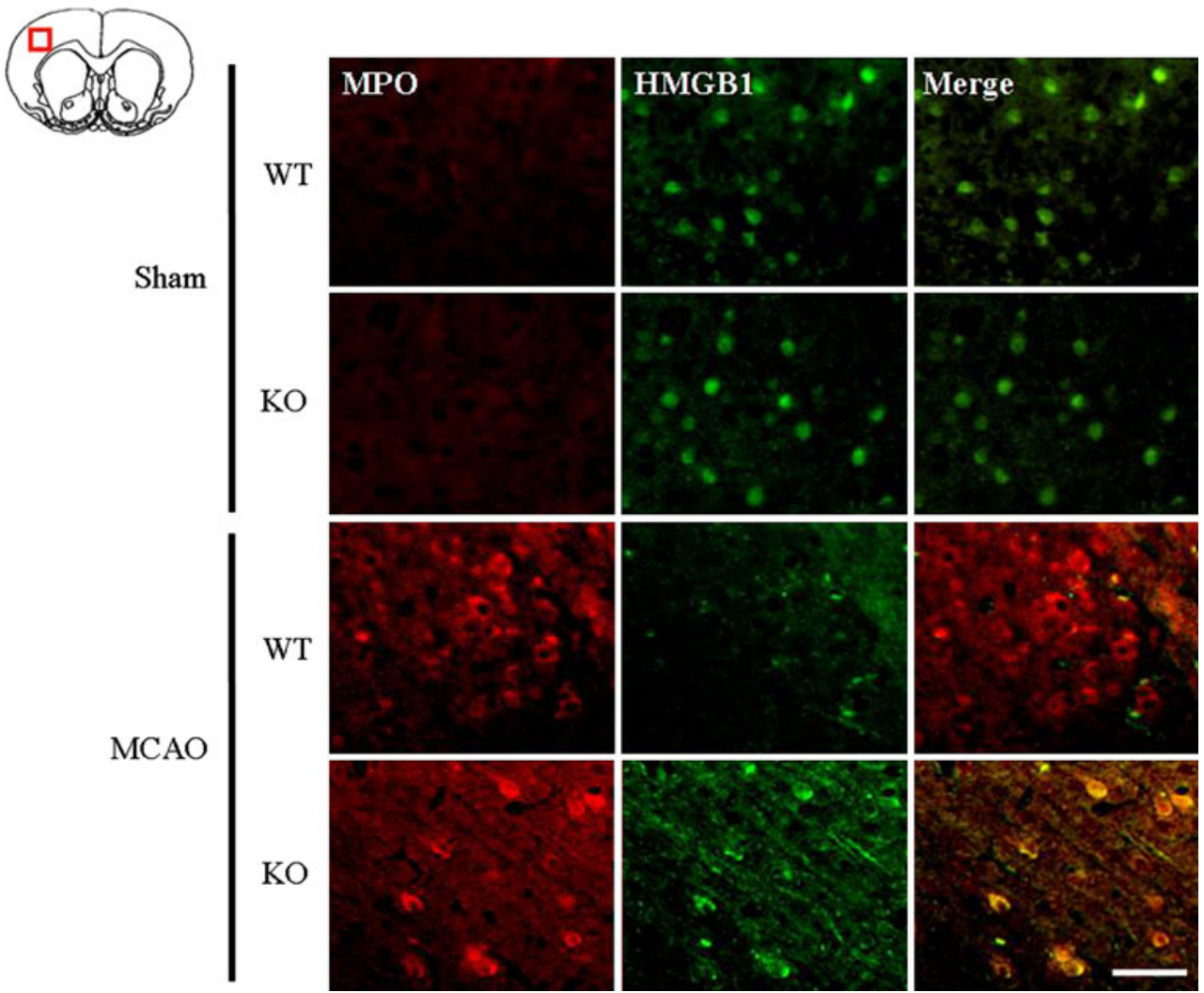

of thrombosis and inflammation. The (UL)VWF-platelet string interacts with leukocytes, and provokes inflammation [5, 7, 37]. The inflammation induces the endothelialULVWF secretion [44]. The proinflammatory cytokines from leukocytes and endothelial cells [such as tumor necrosis factor (TNF)- $\alpha$ and interleukin (IL)-8] stimulate the endothelial ULVWF release and IL-6 protects the ULVWF from cleavage [6]. This would increase the number of ULVWF multimers in plasma sufficiently to aggregate platelets and on vascular endothelial surface to tether platelets and leukocytes onto the endothelium, providing a linkage between thrombosis and inflammation. Of note, HMGB1 stimulates the monocytes/macrophages [3, 34], neutrophils [1, 34] and glial cells [24, 36, 38] to produce TNF- $\alpha$, IL-1, IL-6 and/or IL-8, and incites the microvascular endothelial cells $[12,38]$ to express TNF- $\alpha$, IL- 8 and various adhesion molecules. Namely, the increased plasma HMGB1 in ADAMTS13-deficient mice can upregulate ULVWF, and thus reinforce the association between inflammation and thrombosis. ADAMTS13 may prevent stroke evolution by interfering with the crosstalk between thrombosis and inflammation.

Thrombolytic therapy using tissue plasminogen activator (tPA) for acute stroke has limitations in the therapeutic time window and in the drug dosage due to the risk of hemorrhagic transformation [18]. Further, tPA directly exerts neurotoxicity in the ischemic brain [26]. We suggest that a regulation of the interaction between (UL)VWF-platelet and leukocyte using ADAMTS13 may become a novel therapeutic option in acute brain ischemia. ADAMTS13 does not dissolve the VWF-platelet-primary hemostatic thrombus in the absence of pathologically high fluid shear stress. Therefore, ADAMTS13 may be particularly well suited for acute ischemic stroke without increasing hemorrhagic complications. An early experimental study [48] together with our preliminary data (not shown) demonstrated that recombinant human ADAMTS13 administration reduced infarct volume in stroke model in a VWF-dependent manner without producing cerebral hemorrhage.

This study has several limitations. For example, the reduction of cerebral blood reflow in ADAMTS13KO mice after ischemic insult was continuous and higher than that observed in WT. Therefore, even without the amplified inflammation with HMGB1 neurotoxic effects, only the difference in the blood flow recovery might explain the following more deleterious events in the ischemic brain of ADAMTS13-deficient mice compared to WT. The enhanced elevation of the plasma HMGB1 under ADAMTS13 deficiency after brain ischemia might be also explained simply by the more exacerbated brain damage, regardless of the theoretically intensified interactions between the platelet-(UL)VWF strands and the leukocytes without VWF cleaving protease. The future study required to clarify these issues would include chronological data 
evaluations in the stroke experiments with permanent ischemic procedure (deleting the reperfusion effect) or with enhancing/inhibiting HMGB1 activities by drugs or genetic manipulations.

\section{Conclusions}

A gene deletion of ADAMTS13 renders mice more vulnerable to brain ischemia-reperfusion injury than their wild-type counterparts, when subjected to 30-min MCAO. This preliminary study suggests that ADAMTS13 deficiency may exacerbate systemic and neuronal inflammation after brain ischemia via VWF-dependent pathway, although this remains still hypothetical. Further studies are warranted to better characterize the role of ADAMTS13 in brain ischemia-reperfusion and to provide a novel therapeutic approach for ischemic stroke by regulating VWFdependent inflammation as well as microvascular plugging.

\section{References}

1. Abraham E, Arcaroli J, Carmody A, Wang H, Tracey KJ (2000) HMG-1 as a mediator of acute lung inflammation. J Immunol 165:2950-2954

2. Abumiya T, Fitridge R, Mazur C, Copeland BR, Koziol JA, Tschopp JF, Pierschbacher MD, del Zoppo GJ (2000) Integrin alpha(IIb)beta(3) inhibitor preserves microvascular patency in experimental acute focal cerebral ischemia. Stroke 31:1402-1409

3. Andersson U, Wang H, Palmblad K, Aveberger AC, Bloom O, Erlandsson-Harris H, Janson A, Kokkola R, Zhang M, Yang H, Tracey KJ (2000) High mobility group 1 protein (HMG-1) stimulates proinflammatory cytokine synthesis in human monocytes. J Exp Med 192:565-570

4. Banno F, Kokame K, Okuda T, Honda S, Miyata S, Kato H, Tomiyama Y, Miyata T (2006) Complete deficiency in ADAMTS13 is prothrombotic, but it alone is not sufficient to cause thrombotic thrombocytopenic purpura. Blood 107:3161-3166

5. Bernardo A, Ball C, Nolasco L, Choi H, Moake JL, Dong JF (2005) Platelets adhered to endothelial cell-bound ultra-large von Willebrand factor strings support leukocyte tethering and rolling under high shear stress. J Thromb Haemost 3:562-570

6. Bernardo A, Ball C, Nolasco L, Moake JF, Dong JF (2004) Effects of inflammatory cytokines on the release and cleavage of the endothelial cell-derived ultralarge von Willebrand factor multimers under flow. Blood 104:100-106

7. Chauhan AK, Kisucka J, Brill A, Walsh MT, Scheiflinger F, Wagner DD (2008) ADAMTS13: a new link between thrombosis and inflammation. J Exp Med 205:2065-2074

8. Davi G, Patrono C (2007) Platelet activation and atherothrombosis. N Engl J Med 357:2482-2494

9. Del Zoppo GJ, Schmid-Schonbein GW, Mori E, Copeland BR, Chang CM (1991) Polymorphonuclear leukocytes occlude capillaries following middle cerebral artery occlusion and reperfusion in baboons. Stroke 22:1276-1283

10. Dole VS, Bergmeier W, Mitchell HA, Eichenberger SC, Wagner DD (2005) Activated platelets induce Weibel-Palade-body secretion and leukocyte rolling in vivo: role of P-selectin. Blood 106: 2334-2339
11. Dong JF, Moake JL, Nolasco L, Bernardo A, Arceneaux W, Shrimpton CN, Schade AJ, McIntire LV, Fujikawa K, López JA (2002) ADAMTS-13 rapidly cleaves newly secreted ultralarge von Willebrand factor multimers on the endothelial surface under flowing conditions. Blood 100:4033-4039

12. Fiuza C, Bustin M, Talwar S, Tropea M, Gerstenberger E, Shelhamer JH, Suffredini AF (2003) Inflammation-promoting activity of HMGB1 on human microvascular endothelial cells. Blood 101:2652-2660

13. Fujikawa K, Suzuki H, McMullen B, Chung DP (2001) Purification of human von Willebrand factor-cleaving protease and its identification as a new member of the metalloproteinase family. Blood 98:1662-1666

14. Fujioka M, Hayakawa K, Mishima K, Kunizawa A, Irie K, Higuchi S, Nakano T, Muroi C, Fukushima H, Sugimoto M, Banno F, Kokame K, Miyata T, Fujiwara M, Okuchi K, Nishio K (2010) ADAMTS13 gene deletion aggravates ischemic brain damage: a possible neuroprotective role of ADAMTS13 by ameliorating postischemic hypoperfusion. Blood 115:1650-1653

15. Fujioka M, Taoka T, Matsuo Y, Hiramatsu KI, Sakaki T (1999) Novel brain ischemic change on MRI. Delayed ischemic hyperintensity on T1-weighted images and selective neuronal death in the caudoputamen of rats after brief focal ischemia. Stroke 30:1043-1046

16. Fujioka M, Taoka T, Matsuo Y, Mishima K, Ogoshi K, Kondo Y, Tsuda M, Fujiwara M, Asano T, Sakaki T, Miyasaki A, Park D, Siesjö BK (2003) Magnetic resonance imaging shows delayed ischemic striatal neurodegeneration. Ann Neurol 54:732-747

17. Furlan M, Robles R, Galbusera M, Remuzzi G, Kyrle PA, Brenner B, Krause M, Scharrer I, Aumann V, Mittler U, Solenthaler M, Lammle B (1998) von Willebrand factor-cleaving protease in thrombotic thrombocytopenic purpura and the hemolytic-uremic syndrome. N Engl J Med 339:1578-1584

18. Hacke W, Kaste M, Bluhmki E, Brozman M, Davalos A, Guidetti D, Larrue V, Lees KR, Medeghri Z, Machnig T, Schneider D, von Kummer R, Wahlgren N, Toni D (2008) Thrombolysis with alteplase 3 to 4.5 hours after acute ischemic stroke. N Engl J Med 359: $1317-1329$

19. Hayakawa K, Mishima K, Irie K, Hazekawa M, Mishima S, Fujioka M, Orito K, Egashira N, Katsurabayashi S, Takasaki K, Iwasaki K, Fujiwara M (2008) Cannabidiol prevents a postischemic injury progressively induced by cerebral ischemia via a high-mobility group box1-inhibiting mechanism. Neuropharmacology 55:1280-1286

20. Hayakawa K, Mishima K, Nozako M, Hazekawa M, Irie K, Fujioka M, Orito K, Abe K, Hasebe N, Egashira N, Iwasaki K, Fujiwara M (2007) Delayed treatment with cannabidiol has a cerebroprotective action via a cannabinoid receptor-independent myeloperoxidase-inhibiting mechanism. J Neurochem 102:14881496

21. Hayakawa K, Mishima K, Nozako M, Hazekawa M, Mishima S, Fujioka M, Orito K, Egashira N, Iwasaki K, Fujiwara M (2008) Delayed treatment with minocycline ameliorates neurologic impairment through activated microglia expressing a high-mobility group box1-inhibiting mechanism. Stroke 39:951958

22. Hori O, Brett J, Slattery T, Cao R, Zhang J, Chen JX, Nagashima M, Lundh ER, Vijay S, Nitecki D et al (1995) The receptor for advanced glycation end products (RAGE) is a cellular binding site for amphoterin. Mediation of neurite outgrowth and co-expression of rage and amphoterin in the developing nervous system. J Biol Chem 270:25752-25761

23. Ito I, Fukazawa J, Yoshida M (2007) Post-translational methylation of high mobility group box 1 (HMGB1) causes its cytoplasmic localization in neutrophils. J Biol Chem 282: 16336-16344 
24. Kim JB, Sig Choi J, Yu YM, Nam K, Piao CS, Kim SW, Lee MH, Han PL, Park JS, Lee JK (2006) HMGB1, a novel cytokinelike mediator linking acute neuronal death and delayed neuroinflammation in the postischemic brain. J Neurosci 26:6413-6421

25. Kroll MH, Harris TS, Moake JL, Handin RI, Schafer AI (1991) von Willebrand factor binding to platelet GpIb initiates signals for platelet activation. J Clin Invest 88:1568-1573

26. Liu D, Cheng T, Guo H, Fernandez JA, Griffin JH, Song X, Zlokovic BV (2004) Tissue plasminogen activator neurovascular toxicity is controlled by activated protein C. Nat Med 10: 1379-1383

27. Liu K, Mori S, Takahashi HK, Tomono Y, Wake H, Kanke T, Sato Y, Hiraga N, Adachi N, Yoshino T, Nishibori M (2007) Anti-high mobility group box 1 monoclonal antibody ameliorates brain infarction induced by transient ischemia in rats. FASEB $\mathrm{J}$ 21:3904-3916

28. Lo EH, Dalkara T, Moskowitz MA (2003) Mechanisms, challenges and opportunities in stroke. Nat Rev Neurosci 4:399-415

29. Lotze MT, Tracey KJ (2005) High-mobility group box 1 protein (HMGB1): nuclear weapon in the immune arsenal. Nat Rev Immunol 5:331-342

30. Moake JL, Rudy CK, Troll JH, Weinstein MJ, Colannino NM, Azocar J, Seder RH, Hong SL, Deykin D (1982) Unusually large plasma factor VIII: von Willebrand factor multimers in chronic relapsing thrombotic thrombocytopenic purpura. N Engl J Med 307:1432-1435

31. Mori E, del Zoppo GJ, Chambers JD, Copeland BR, Arfors KE (1992) Inhibition of polymorphonuclear leukocyte adherence suppresses no-reflow after focal cerebral ischemia in baboons. Stroke 23:712-718

32. Mori T, Town T, Tan J, Tateishi N, Asano T (2005) Modulation of astrocytic activation by arundic acid (ONO-2506) mitigates detrimental effects of the apolipoprotein E4 isoform after permanent focal ischemia in apolipoprotein $\mathrm{E}$ knock-in mice. J Cereb Blood Flow Metab 25:748-762

33. Muhammad S, Barakat W, Stoyanov S, Murikinati S, Yang H, Tracey KJ, Bendszus M, Rossetti G, Nawroth PP, Bierhaus A, Schwaninger M (2008) The HMGB1 receptor RAGE mediates ischemic brain damage. J Neurosci 28:12023-12031

34. Park JS, Svetkauskaite D, He Q, Kim JY, Strassheim D, Ishizaka A, Abraham E (2004) Involvement of toll-like receptors 2 and 4 in cellular activation by high mobility group box 1 protein. J Biol Chem 279:7370-7377

35. Passalacqua M, Patrone M, Picotti GB, Del Rio M, Sparatore B, Melloni E, Pontremoli S (1998) Stimulated astrocytes release high-mobility group 1 protein, an inducer of LAN-5 neuroblastoma cell differentiation. Neuroscience 82:1021-1028
36. Pedrazzi M, Patrone M, Passalacqua M, Ranzato E, Colamassaro D, Sparatore B, Pontremoli S, Melloni E (2007) Selective proinflammatory activation of astrocytes by high-mobility group box 1 protein signaling. J Immunol 179:8525-8532

37. Pendu R, Terraube V, Christophe OD, Gahmberg CG, de Groot PG, Lenting PJ, Denis CV (2006) P-selectin glycoprotein ligand 1 and beta2-integrins cooperate in the adhesion of leukocytes to von Willebrand factor. Blood 108:3746-3752

38. Qiu J, Nishimura M, Wang Y, Sims JR, Qiu S, Savitz SI, Salomone S, Moskowitz MA (2008) Early release of HMGB-1 from neurons after the onset of brain ischemia. J Cereb Blood Flow Metab 28:927-938

39. Rouhiainen A, Imai S, Rauvala H, Parkkinen J (2000) Occurrence of amphoterin (HMG1) as an endogenous protein of human platelets that is exported to the cell surface upon platelet activation. Thromb Haemost 84:1087-1094

40. Ruggeri ZM (2007) The role of von Willebrand factor in thrombus formation. Thromb Res 120(Suppl 1):S5-S9

41. Sadler JE (2008) Von Willebrand factor, ADAMTS13, and thrombotic thrombocytopenic purpura. Blood 112:11-18

42. Scaffidi P, Misteli T, Bianchi ME (2002) Release of chromatin protein HMGB1 by necrotic cells triggers inflammation. Nature 418:191-195

43. Siedlecki CA, Lestini BJ, Kottke-Marchant KK, Eppell SJ, Wilson DL, Marchant RE (1996) Shear-dependent changes in the three-dimensional structure of human von Willebrand factor. Blood 88:2939-2950

44. Vischer UM (2006) von Willebrand factor, endothelial dysfunction, and cardiovascular disease. J Thromb Haemost 4:1186-1193

45. Wang H, Bloom O, Zhang M, Vishnubhakat JM, Ombrellino M, Che J, Frazier A, Yang H, Ivanova S, Borovikova L, Manogue KR, Faist E, Abraham E, Andersson J, Andersson U, Molina PE, Abumrad NN, Sama A, Tracey KJ (1999) HMG-1 as a late mediator of endotoxin lethality in mice. Science 285:248-251

46. Yin H, Liu J, Li Z, Berndt MC, Lowell CA, Du X (2008) Src family tyrosine kinase Lyn mediates VWF/GPIb-IX-induced platelet activation via the cGMP signaling pathway. Blood 112: 1139-1146

47. Zhang J, Takahashi HK, Liu K, Wake H, Liu R, Maruo T, Date I, Yoshino T, Ohtsuka A, Mori S, Nishibori M (2011) Anti-high mobility group box-1 monoclonal antibody protects the bloodbrain barrier from ischemia-induced disruption in rats. Stroke 42:1420-1428

48. Zhao BQ, Chauhan AK, Canault M, Patten IS, Yang JJ, Dockal M, Scheiflinger F, Wagner DD (2009) von Willebrand factorcleaving protease ADAMTS13 reduces ischemic brain injury in experimental stroke. Blood 114:3329-3334 